\title{
Optimized xylose isomerase uptake and expression level in Saccharomyces cerevisiae for improving ethanol production
}

\author{
Mei Zhang ${ }^{1}$, Wen-Jing Fan ${ }^{1}$, Jing-Yu Wang ${ }^{2 *}$, Li-Min Cao $^{1 *}$ \\ ${ }^{1}$ College of Life Science, Capital Normal University, Haidian District, Beijing 100048, China \\ ${ }^{2}$ Department of Chemical Engineering and Materials Science, University of Minnesota, Twin Cities, Minneapolis, Minnesota, USA
}

\begin{abstract}
The ability to engineer the yeast Saccharomyces cerevisiae to efficiently convert lignocellulosic biomass to ethanol remains a considerable challenge. Here, we propose a new reprogrammable strategy to optimize the expression level of the xylose isomerase $(X I)$ gene with the induction of mutations in S. cerevisiae to improve efficient ethanol production and productivity. We sought to fine-tune the xylose uptake and catabolism abilities of $S$. cerevisiae during fermentation by improving efficiency of the xylose transporter, which was fused with four copies of the $X I$ gene under the control of different promoters to obtain recombinant yeast strains. In fermentation experiments, the optimized strain CW9 cultured in yeast extract-peptone (YP) medium containing approximately $65 \mathrm{~g} / \mathrm{L}$ glucose and $55 \mathrm{~g} / \mathrm{L}$ xylose produced consistent ethanol yields of $0.45 \mathrm{~g} / \mathrm{g}$ total sugar in about $72 \mathrm{~h}$, which was close to $90 \%$ of the theoretical yield. These promising results indicate that strain CW9 is the best producer of ethanol from mixed sugar when synthetically regulating the xylose assimilation pathway. Overall, this study provides an optimal method to control XI expression levels to find better conditions for enhancing biofuel production.

Keywords: xylose; promoter elements; transporter; ethanol; Saccharomyces cerevisiae
\end{abstract}

* Correspondence to:

Li-Min Cao, College of Life Science, Capital Normal University, Haidian District, Beijing 100048, China; E-mail: caolimin@cnu.edu.cn

Department of Chemical Engineering and Materials Science, University of Minnesota, Twin Cities, Minneapolis, Minnesota, USA; E-mail: wang4968@umn.edu

\section{Received: January 17, 2018; Accepted: February 7, 2018; Published Online: March 26, 2018}

Citation: Zhang, M., Fan, W.J., Wang, J.Y. and Cao, L.M., 2018, Optimized xylose isomerase uptake and expression level in Saccharomyces cerevisiae for improving ethanol production. Applied Environmental Biotechnology, 3(1), 44-52. http://doi.org/10.26789/AEB.2018.01.007.

Copyright: Optimized xylose isomerase uptake and expression level in Saccharomyces cerevisiae for improving ethanol production. (C) 2018 Mei Zhang et al.. This is an Open Access article distributed under the terms of the Creative Commons Attribution-Noncommercial 4.0 International License, permitting all non-commercial use, distribution, and reproduction in any medium, provided the original work is properly cited and acknowledged.

\section{Introduction}

The cost-effective production of second-generation bioethanol requires efficient utilization of the most abundant glucose source to yield a significant fraction of the xylose obtained after hydrolysis of certain types of lignocellulosic biomass. The yeast Saccharomyces cerevisiae, which has been traditionally used for industrial ethanol production owing to its high rate of glucose fermentation, shows high tolerance to ethanol, inhibitors, and other industrial process conditions, and does not efficiently metabolize pentose sugars, particularly xylose. Therefore, numerous xylose-utilizing $S$. cerevisiae strains have been established using intensive research and genetic engineering approaches to efficiently utilize xylose, resulting in higher overall ethanol yield and productivity (Bengtsson et al., 2009; Olofsson et al., 2011).

Xylose assimilation by different $S$. cerevisiae strains differs only with respect to the pathways used for xylose: the oxidoreductive pathway with the NAD(P)H-dependent xylose reductase (XR) and the $\mathrm{NAD}^{+}$-dependent xylitol dehydrogenase (XDH) from Scheffersomyces stipitis (Johansson et al., 2001; Hou et al., 2007), or the isomerization pathway with xylose isomerase $(X I)$ from fungal and bacterial genes (Brat et al., 2009; Madhavan et al., 2009). However, an imbalance in the NADH/NADPH cofactor ratio in the $\mathrm{XR}$ and $\mathrm{XDH}$ pathways can result in variable production levels of xylitol, and therefore reduce ethanol yields. Some recombinant strains were constructed by chromosomal integration of codon-optimized prokaryotic XI from Clostridium phytofermentans in the xylose utilization pathway (Brat et al., 2009; Demeke et al., 2013). Furthermore, the XI pathway in the fungus Piromyces sp., which shows higher activity, could functionally enable yeast cells to ferment xylose efficiently (Kuyper et al., 2015). Nevertheless, this XI activity requires further evolution or adaptation in the recombinant strain to allow for anaerobic growth on xylose (Lee et al., 2012; Zhou et al., 2012). Furthermore, overexpression of the rate-controlling xylulokinase $(X K)$ gene (Eliasson et al., 2000) and the genes TAL1, TKL1, RPE1, and RKI1 involved in the non-oxidative part of the pentose phosphate pathway (PPP) have been reported to be beneficial for improving xylose uptake, in addition to the overexpression of uptake systems for transporting xylose (Young et al., 2012; Kuyper et al., 2015). Thus, xylose utilization in an S. cerevisiae strain expressing Piromyces XI has been successfully improved with the use of combined metabolic and evolutionary engineering strategies.

However, the co-fermentation of xylose and glucose remains a challenge, and yeast can generally only utilize xylose 
after depletion of glucose owing to glucose repression during the uptake of xylose into the cells. This is because xylose is taken up by $S$. cerevisiae cells via the glucose uptake systems (Saloheimo et al., 2007), and xylose uptake is competitively inhibited by glucose because of preference of the transporters for glucose. To date, some specific xylose transporters have been functionally expressed in yeast, which could specifically mediate the uptake of xylose into $S$. cerevisiae cells to some degree but were not able to effectively transport hexoses or were still somewhat inhibited by glucose (Subtil et al., 2012). Recently, the mutant hexose transporter HXT7 (F79S), which was established through a laboratory evolution regime, was reported to show increased xylose uptake rates that allowed the $S$. cerevisiae strain to grow rapidly with xylose, and even under mixed glucose and xylose conditions (Apel et al., 2016). In addition, substitution of the $\mathrm{N}$-terminal lysine residues in the endogenous hexose transporters HXT1 and HXT36, which function as specific xylose transporters that are subjected to catabolite degradation, resulted in their improved retention at the cytoplasmic membrane in the absence of glucose, leading to improved xylose fermentation (Nijland et al., 2016). An artificial complex between an endogenous sugar transporter and a heterologous xylose isomerase in $S$. cerevisiae resulted in accelerated xylose consumption and substantially diminished xylitol production, with a concomitant increase in the production of ethanol (Thomik et al., 2017). Therefore, increasing bioethanol production during mixed glucose and xylose fermentation through efficient modification of the xylose utilization pathway in $S$. cerevisiae strains after consuming sufficient amounts of glucose to support yeast cell growth may require the following conditions: increasing the expression levels and activities of heterologous proteins, regulating the redox balance, realizing a proper metabolic burden, and accumulation of less toxic intermediates (Salis, et al., 2009; Bond-Watts et al., 2011).

Several promoters showing strong activity based on our test libraries were previously used to express the key enzymes $X I$ and $X K$ to optimize xylose metabolic pathways, resulting in improved xylose utilization (Yuan et al., 2017). Based on this background, we used these same promoters in the present study to evaluate the effect of genetic modifications on improving xylose utilization in $S$. cerevisiae strains expressing the $X I$ pathway. First, we constructed two yeast multi-copy expression vectors harboring four non-oxidative PPP genes, which were transformed into the xylose assimilation pathway. Second, we introduced two promoters that show strong activity, $p P G K 1$ and $p H X T 7$, capable of optimizing high expression levels of mutated $X I$ and $X K$ in the cytoplasm of the yeast four-gene strain so as to tailor the XI pathway toward obtaining a higher ethanol yield. Third, we designed novel fusion proteins using xylose-transporting hexose transporters, including hexogenous $H X T 7$ and heterogeneous $G X S 1$, with a mutated $X I$ gene to improve the xylose uptake rate in the cell membrane in combination with the cytoplasmic $X I$ strains mentioned above. As a supplementary molecule, the addition of the endogenous transporter HXT7 may ensure the proper uptake of xylose and glucose. The characteristics of the resulting recombinant $S$. cerevisiae strains with higher ethanol production were studied to systematically determine the fac- tors affecting the metabolic pathway flux of $X I$, such as copy number variations and different cell locations. The optimized yeast XI strain (designated as strain CW9) showed an ethanol yield that was nearly $90 \%$ of the theoretical maximum within about $72 \mathrm{~h}$.

\section{Materials and Methods}

\subsection{Yeast strains, Plasmids, and Media}

In this study, the $S$. cerevisiae haploid strains used were derived from the diploid industrial strain YC-DM (Angel Yeast, China). The Scheffersomyces stipitis genomic DNA was extracted from Sch. stipitis CBS6054 (Jeffries et al., 2007). Yeast cells were propagated in $2 \%$ YPD medium (yeast extract, $10 \mathrm{~g} / \mathrm{L}$; peptone, $20 \mathrm{~g} / \mathrm{L}$; glucose, $20 \mathrm{~g} / \mathrm{L}$ ). YNB medium without amino acids (Difco) supplemented with $2 \%(\mathrm{w} / \mathrm{v})$ glucose and certain amino acids (according to the requirement of each strain), and 5-fluoroorotic acid medium (5FOA) were used for yeast growth and the screening of correct transformants. The yeast strains, plasmids, and primers used in this study are listed in Tables 1, 2, and 3, respectively. S. cerevisiae strains were maintained at $80^{\circ} \mathrm{C}$ in a stock medium composed of YP and 20\% glycerol. The methods and physical map for the construction of the four plasmids pCYTO1-XI, pCYTO2-XI, pPMHXT7-XI, and pPM-GXS1-XI are provided in the Supplementary Materials and in Supplementary Figures S1-S4. We adopted the yeast codon-optimized version of the mutated XI gene (see the Supplementary Materials).

Table 1.Plasmids used in this study.

\begin{tabular}{llc}
\hline Plasmids & Marker and description & Reference \\
\hline pUC18-RKUR & $A M P$, & Kong et al., 2017 \\
pBluescript SKP & $A M P$ & Stratagene, La Jolla, CA \\
& $A M P$, PGK1p-RKI1-CYC1t/ADH1p-TAL1-ADH1t/ & Xiong et al., 2011 \\
5-Final & PGK1p-RPE1-CYC1t/ADH1p-TKL1-ADH1t & \\
& $A M P$, ADH1p-XR-ADH1t/PGK1p-XDH-PGK1t/ & Xiong et al., 2011 \\
pGU-3X & PGK1p-XK-PGK1t & \\
pCYTO1-XI & $A M P$, PGK1p-XI ${ }^{6 m}$-PGK1t/PGK1p-XK-XKt & This work \\
pCYTO2-XI & $A M P$, HXT7p-XI ${ }^{6 m}$-PGK1t/PGK1p-XK-XKt & This work \\
pPM-HXT7-XI & $A M P$, PGK1p-HXT7(ORF)-XI ${ }^{6 \mathrm{~m}}$-PGK1t/PGK1p-XK- & This work \\
pPM-GXS1-XI & $A M P$, PGK1p-GXS1 ${ }^{1 \mathrm{~m}}$-XI ${ }^{6 \mathrm{~m}}$-PGK1t/PGK1p-XK-XKt & This work \\
\hline
\end{tabular}

\subsection{Construction of Yeast XI Strains}

To overexpress the four genes RPE1, RKI1, TAL1, and TKL1, the plasmid "5-final", carrying a selective marker of the G418 kit, was digested with $\mathrm{HpaI}$. The digested fragment was used to transform the yeast $S$. cerevisiae haploid strain to obtain strain $\mathrm{CW} 1$ with all four genes integrated (Xiong et al., 2011).

The plasmids pCYTO1-XI and pCYTO2-XI, containing two genes for $X I$ and $X K$, were digested with $A s c \mathrm{I}$ and $X m a \mathrm{I}$ and then transformed into strain $\mathrm{CW} 1$, creating the resulting yeast strains $\mathrm{CW} 2$ and CW3, respectively. Moreover, the plasmids pPM-GXS1XI with a fusion protein of $G X S 1$ and XI, and pPM-HXT7-XI containing a fusion protein of $H X T 7$ and $X I$ were digested by AscI-SacI and then transformed into the strain $\mathrm{CW} 1$, creating the resulting engineered strains CW4 and CW5, respectively. Moreover, the engineered strain CW6 was obtained by introducing pCYTO2-XI 
Table 2.Strains used in this study.

\begin{tabular}{llc}
\hline Strains & Genotypes & References \\
\hline YC-DM & MAT a/ $\alpha$ & Angel Yeast, China \\
CBS6054 & Sch. stipitis CBS 6054 & Jeffries et al., 2007 \\
WT & MATa ura 3 & This work \\
CW1 & WT, 5-Final & This work \\
CW2 & CW1, ura $::$ pCYTO1-XI & This work \\
CW3 & CW1, ura $::$ pCYTO2-XI & This work \\
CW4 & CW1, ura $::$ pPM-HXT7-XI & This work \\
CW5 & CW1, ura3 :: pPM-GXS1-XI & This work \\
CW6 & CW2, ura $::$ pCYTO1-XI:: pCYTO2-XI & This work \\
CW7 & CW4, ura $::$ pPM-GXS1-XI & This work \\
CW8 & CW4, ura $::$ pPM-HXT7-XI & This work \\
CW9 & CW4, ura $::$ pPM-HXT7-XI:: pPM-GXS1-XI & This work \\
\hline
\end{tabular}

into strain CW2 (Table 2). The yeast transformants were isolated on minimal medium lacking uracil and were verified for the correct integration of the RKUR cassette with diagnostic polymerase chain reaction. The isolates showing overexpression of the targeted gene were incubated on FOA plates to select a loop-out of the URA3 gene through homologous recombination between the repeated sequences flanking the $U R A 3$ gene in the integration cassette (Kong et al., 2007; Xiong et al., 2011).

Moreover, the plasmid pPM-GXS1-XI or pPM-HXT7-XI previously digested by AscI-SacI was used for transformation of the target strain CW6, creating the resulting engineered strain CW7 or CW8, respectively. At the same time, the engineered strain CW9 was obtained by introducing pPM-HXT7-XI into strain CW7 (Table 2). Isolation and verification of the transformants and subsequent loop-out of the vector sequence containing the URA3 gene were performed essentially as described above.

\subsection{Fermentation Experiments}

The recombinant yeast strains were first cultivated aerobically in $2 \%$ YPD medium overnight at $30^{\circ} \mathrm{C}$. The overnight-grown cells were centrifuged at $3000 \mathrm{rpm}$ and $4^{\circ} \mathrm{C}$ and then washed twice with sterile water. Yeast cells were transferred to $250-\mathrm{mL}$ shake flasks with 100 $\mathrm{mL}$ of YP medium containing $55 \mathrm{~g} / \mathrm{L}$ xylose and $65 \mathrm{~g} / \mathrm{L}$ glucose to ferment at $30^{\circ} \mathrm{C}$ with continuous shaking at $200 \mathrm{rpm}$. Initial cell densities were adjusted to an optical density at $600 \mathrm{~nm}$ of 0.2 . All flasks were sealed with sealing film (Parafilm M, USA) after the yeast cells were harvested. The cultures were initially aerobic and then gradually became oxygen-limited as cultivation proceeded. All of the flask fermentation experiments were performed in triplicate, and less than 5\% variation was observed between independent fermentations.

\subsection{Analysis of Substrates and Metabolites}

Concentrations of substrates and metabolites in the culture broth, including glucose, xylose, xylitol, and ethanol, were analyzed using high-performance liquid chromatography (HPLC, Agilent Technologies 1260 Series USA), containing an Aminex HPX-87 H ion exchange column (BioRad, USA) and a refractive index detector (Waters 2414 RI detector). Samples were centrifuged in 2-mL microcentrifuge tubes at $14,000 \mathrm{rpm}$ for $5 \mathrm{~min}$. The supernatant was filtered using $0.2-\mu \mathrm{m}$ filters, and the filtered samples were stored at $-20^{\circ} \mathrm{C}$. The mobile phase was $5 \mathrm{mM} \mathrm{H}_{2} \mathrm{SO}_{4}$ at a flow rate of 0.6
$\mathrm{mL} / \mathrm{min}$. The column temperature and detection temperature were maintained at $30^{\circ} \mathrm{C}$ and $50^{\circ} \mathrm{C}$, respectively.

\subsection{Determination of Specific $X I$ Activity}

The specific $X I$ activity from cell extracts was assayed using a modified method (Zhou et al., 2012). The reaction mixture $(1 \mathrm{~mL})$ contained $100 \mathrm{mM}$ Tris- $\mathrm{HCl}$ buffer $(\mathrm{pH} 7.5)$, $0.2 \mathrm{mM} \mathrm{NADH}, 10 \mathrm{mM} \mathrm{MgCl}$, and $2 \mathrm{U}$ sorbitol dehydrogenase (Roche, Mannheim, Germany), as well as $20 \mathrm{~mL}$ of equilibrated fresh cell extract at $30^{\circ} \mathrm{C}$ for $5 \mathrm{~min}$. The protein contents of the cell extracts were determined using the Pierce $660 \mathrm{~nm}$ Protein Assay kit (Thermo Scientific). The reaction was started by the addition of xylose to a final concentration of $500 \mathrm{mM}$. A molar extinction coefficient of $6.25 \mathrm{mM}^{-1}$ $\mathrm{cm}^{-1}$ at $340 \mathrm{~nm}$ for NADH was used to calculate the specific $X I$ activity, expressed as units per milligram of protein. One unit corresponds to the conversion of $1 \mu \mathrm{M}$ of xylose into xylulose per minute under the specified assay conditions.

\subsection{Directed Evolution}

All of the engineered XI strains obtained were used to initiate a directed evolution procedure to improve the xylose utilization rate and ethanol production, which consisted of the same three stages according to a recent evolution method (Zhou et al., 2012). In brief, the cells were first grown aerobically in YPX medium through sequential batch cultivation. After about 70 generations of evolution with the same dry cell weight, the isolated single strain displayed improved aerobic growth. Further evolution of the cell was carried out using the same sequential batch cultivation setup in YNBX medium from a micro-aerobic condition to anaerobic condition. The resulting evolved strain should show improved anaerobic growth. At the third stage, evolution of the whole strain was performed under continuous anaerobic cultivation using a xylose gradient as the limiting substrate.

\section{Results and Discussion}

\subsection{Enhanced XI Activities for Key XI Strains}

A previous study showed that metabolic/evolutionary engineering for improved growth on xylose in a yeast strain led to increased activity of the heterologous Piromyces XI gene expressed from a plasmid (Brat et al., 2009). To test whether a similar change might have occurred in our strains, we measured $X I$ activity in cell extracts of the key XI strains constructed: CW2, CW6, and CW9. The specific XI activity in CW9 and CW6 was 3- and 2-fold higher than that of the precursor strain CW2, respectively (Figure 1). This marked improvement in $X I$ activity, up to $0.55 \mathrm{U} / \mathrm{mg}$ protein, could be a crucial reason for the improved xylose fermentation rate observed in the CW6 or CW9 strain, which is consistent with the results of previous similar reports for evolutionarily engineered strains (Shen et al., 2012; Zhou et al., 2012; 
Table 3.Primers used in this study.

\begin{tabular}{|c|c|}
\hline Primer name & Sequence \\
\hline $\mathrm{ADH} 2 \mathrm{p}-\mathrm{F}$ & 5'-AAACCCGGTACCGGCGCGCCAGGGATTTTGTCTTCATTAA \\
\hline $\mathrm{ADH} 2 \mathrm{p}-\mathrm{R}$ & 5'-GGGCCC CTCGAGGTTTAAAC TGTGTATTACGATATAGTTA \\
\hline PGK1p-F1 & 5'-AAACCC GTTTAAAC AGGCATTTGCAAGAATTACT \\
\hline PGK1p-R1 & 5'-GGGCCC GTCGACCATATG TGTTTTATATTTGTTGTAAAAAG \\
\hline XI-F & 5'-AAATTT GTCGAC ATGGCTAAGGAATACTTCCC \\
\hline XI-R & 5'-GGGCCC ATCGATTTAATTAA TTATTGGTACATAGCGATGA \\
\hline PGK1t-F & 5'-GGGCCC TTAATTAA ATTGAATTGAATTGAAATCG \\
\hline PGK1t-R & 5' -AAACCC GCTAGC GTTGCAAGTGGGATGAGCTT \\
\hline RUR-F & 5'- GGGCCC GCTAGC GAGCAGCATAAACGACTGCT \\
\hline RUR-R & 5'- GGGCCC CTGCAG ACGCTCAATGTTGTTCATGA \\
\hline PGK1p-F2 & 5'- ATATAT CTGCAG AGGCATTTGCAAGAATTACT \\
\hline PGK1p-R2 & 5'- GGGCCC GGATCC TTTGAATATGTATTACTTGG \\
\hline $\mathrm{ADH} 2 \mathrm{t}-\mathrm{F}$ & 5'- ATGCAT GCGGCCGC TGTTTTATATTTGTTGTAAAAAG \\
\hline $\mathrm{ADH} 2 \mathrm{t}-\mathrm{R}$ & 5'- GGATCC GAGCTCCCCGGG GTATAGAATTATATAACTTG \\
\hline XK-F & 5'- CCCTTT GGATCC ATGTTGTGTTCAGTAATTCA \\
\hline XK-R & 5'- CCCGGG GCGGCCGC ACTCTTCATTTATTGAATTTT \\
\hline CRE1 p-F & 5' -AAATTT GGCGCGCC TTGACTGTAATTGCCGAATA \\
\hline CRE1 p-R & 5' - GGGCCC GTTTAAAC GCCAATGAATGAATTTTGC \\
\hline Cre1t-F & 5' -ATATAT GCGGCCGC TGTGCTGCAAAATTTTCCAG \\
\hline Cre1t-R & 5' -CCCAAA_CCCGGG CTGTTGAGTTGGCGTATGCA \\
\hline HXT7 p-F & $5{ }^{\prime}$-GGGCCC GTTTAAAC TCTAGTTTCTGCCTTAAACA \\
\hline HXT7 p-F & 5' -GGGCCC CATATG TTTTTGATTAAAATTAAAAA \\
\hline FPS1p-F & 5'- AAACCC GGCGCGCC TCCATATATGTTAAAATGCT \\
\hline FPS1p-R & 5'- CCCGGG GTTTAAAC GTCTTTTAATGCATTAGAATGT \\
\hline FPS1t-F & 5'- CCCTTT GCGGCCGC GAAAACAGACAAGAAAAAGA \\
\hline FPS1t-R & 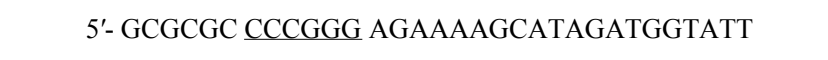 \\
\hline HXT7O-F & 5'- GGGCCC CATATGATGTCACAAGACGCTGCTAT \\
\hline HXT7-XI-R & 5'- GAAGTATTCCTTAGCCATTTTGGTGCTGAACATTCTCT \\
\hline XI-HXT7-F & 5'- AGAGAATGTTCAGCACCAAAATGGCTAAGGAATACTTC \\
\hline XIO-R & 5'- GGGCCC TTAATTAA TTATTGGTACATAGCGATGA \\
\hline PGK1p-F3 & 5'- GGGCCC $\underline{\text { CTGCAG AGGCATTTGCAAGAATTACT }}$ \\
\hline PGK1p-R3 & 5'- GGGCCC GGATCC TGTTTTATATTTGTTGTAAAAAG \\
\hline GXS1O-F & 5'- GGGCCC GGATCC ATGGGTTTGGAGGACAATAGA \\
\hline GXS1-XI-R & 5'- GGGAAGTATTCCTTAGCCATAACAGAAGCTTCTTCAGACA \\
\hline XI-GXF1-F & 5'- TGTCTGAAGAAGCTTCTGTTATGGCTAAGGAATACTTCCC \\
\hline XIO-R2 & 5'- AAATTT CCCGGG TTATTGGTACATAGCGATGA \\
\hline AU-F & 5'- GCGCGC CCCGGG GCGAATTTCTTATGATTTAT \\
\hline AU-R & 5'- AAATTT GATATC CACATGTGTTTTTAGTAAAC \\
\hline
\end{tabular}




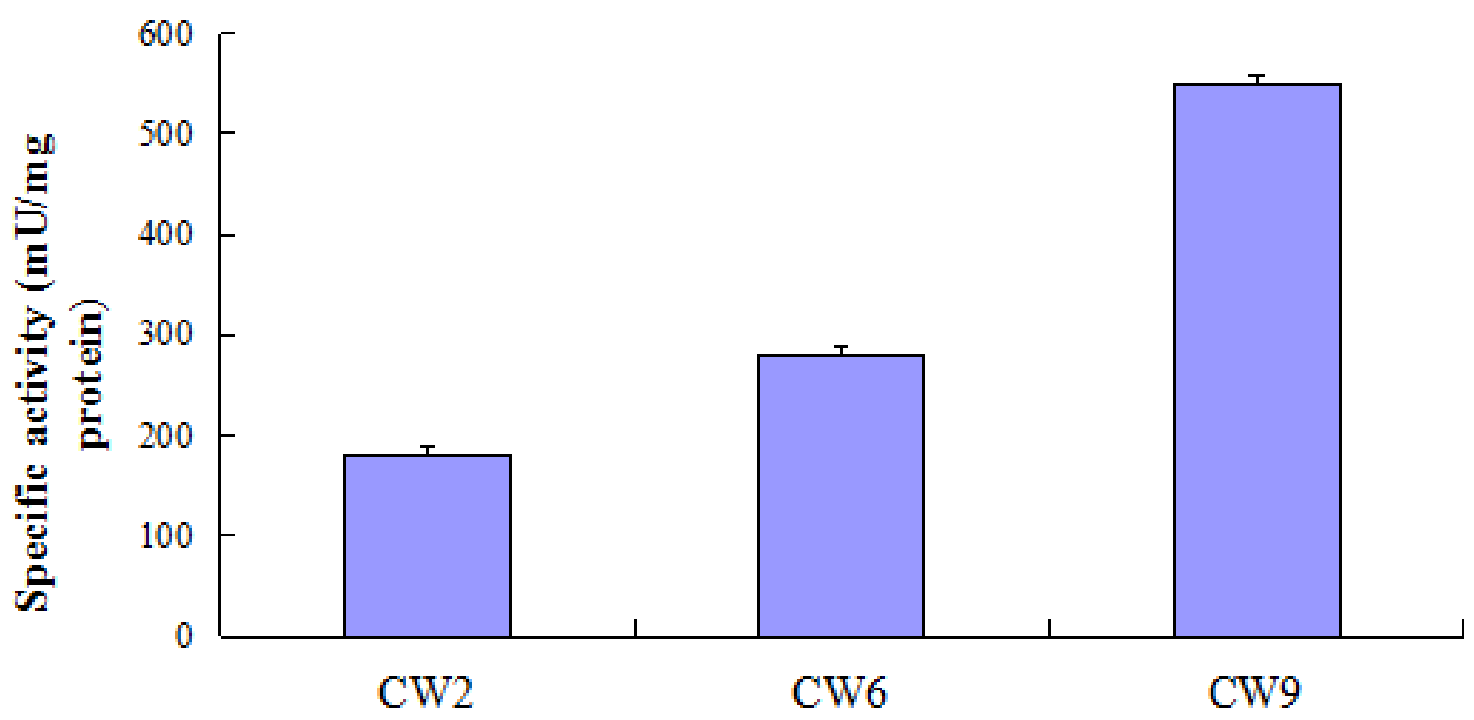

Figure 1. $X I$ activities of three $X I$ strains. The specific $X I$ activities were measured in the cell extracts of strains CW2, CW6, and CW9. Error bars represent the standard deviations from triplicate experiments.

Demeke et al., 2013). These results confirmed that increased copy numbers of XI genes contribute to improved xylose utilization in the evolved strains by integration into the yeast genome. This further confirms that high $X I$ activity is indeed beneficial for improving the xylose fermentation rate, but that achieving this advantage requires the introduction of six mutations in the genome of a given strain.

\subsection{Fermentation Profile from Mixed Sugars for Engineered Strains with XI Located in the Cell Cytoplasm}

We combined different promoters based on the expression of the yeast codon-optimized version of mutated XI (see Supplementary Materials) with an evolutionary engineering approach, and developed five industrial $S$. cerevisiae strains that could efficiently convert xylose to ethanol with a rational yield and productivity level. The fermentation profiles of the five strains (CW2-6) obtained through sequential metabolic/evolutionary engineering were evaluated in YPXD medium containing $65 \mathrm{~g} / \mathrm{L}$ glucose and $55 \mathrm{~g} / \mathrm{L}$ xylose (Figure 2). Strains CW2, CW3, and CW6 consumed 100\% glucose and $39.5 \mathrm{~g} / \mathrm{L}(72.5 \%), 42.2 \mathrm{~g} / \mathrm{L}(74.4 \%)$, and 44.9 $\mathrm{g} / \mathrm{L}(82.4 \%)$ xylose after $48 \mathrm{~h}$, and produced $36.7 \mathrm{~g} / \mathrm{L}, 41.0$ $\mathrm{g} / \mathrm{L}$, and $44.2 \mathrm{~g} / \mathrm{L}$ ethanol with $7 \mathrm{~g} / \mathrm{L}, 5.1 \mathrm{~g} / \mathrm{L}$, and $3.8 \mathrm{~g} / \mathrm{L}$ of xylitol formation, respectively. After $84 \mathrm{~h}, \mathrm{CW} 2$ consumed $97.6 \%$ xylose and produced a maximum ethanol concentration of $42.2 \mathrm{~g} / \mathrm{L}$ with $7.8 \mathrm{~g} / \mathrm{L}$ xylitol (Figure 2A). However, CW3 and CW6 consumed $96.1 \%$ and $97.6 \%$ xylose and produced a maximum ethanol concentration of $45.5 \mathrm{~g} / \mathrm{L}$ and 47.9 $\mathrm{g} / \mathrm{L}$ with $6.1 \mathrm{~g} / \mathrm{L}$ and $4.7 \mathrm{~g} / \mathrm{L}$ of xylitol, respectively, after 72 $\mathrm{h}$ (Figure $2 \mathrm{~B}$ and 2E). The yields of ethanol and xylitol from total sugar for the three strains (CW2, 3, 6) were $0.35 \mathrm{~g} / \mathrm{g}$, $0.38 \mathrm{~g} / \mathrm{g}$, and $0.40 \mathrm{~g} / \mathrm{g}$, and $0.07 \mathrm{~g} / \mathrm{g}, 0.05 \mathrm{~g} / \mathrm{g}$, and $0.04 \mathrm{~g} / \mathrm{g}$, respectively. Strains CW2 and CW3 had very similar maximum sugar consumption rates and ethanol production rates under anaerobic conditions. During this fermentation process, the strains CW4 and CW5 showed maximum ethanol production of $45.8 \mathrm{~g} / \mathrm{L}$ and $46.5 \mathrm{~g} / \mathrm{L}$ with $4.3 \mathrm{~g} / \mathrm{L}$ and 2.2 $\mathrm{g} / \mathrm{L}$ of xylitol within $72 \mathrm{~h}$, respectively (Figure 2C and 2D). Their xylose utilization capabilities displayed a slightly improved fermentation profile compared to those of CW2 or CW3 (Figure 2). Therefore, improving the flux of xylose into the cell via fusing the expressed transporter with the XI gene could help to achieve more efficient uptake for metabolism. These results indicate that our experimental design resulted in an improved impact of the fusion protein for the relative activity of strains CW4 and CW5. In brief, strain CW6 with two copies of $X I$ showed slightly improved fermentation performance compared to that of the other four strains. The co-fermentation comparisons from a sugar mixture for the five $X I$ strains are summarized in Table 4.

CW3 showed slightly higher ethanol production and reduced xylitol formation compared to CW2, which was due to the moderate $X I$ expression level from the promoter of the xylose-transporting hexose transporter $H X T 7$ for CW3 grown in mixed sugar compared with the promoter of $P G K 1$. Fusing the expressed transporter with the XI gene for CW4 and CW5 resulted in more efficient xylose utilization, suggesting a potential advantage of expressing a fusion protein compared to expressing the same copy number of the $X I$ and transporter genes. The higher ethanol-producing capability in CW6 could be explained by the fact that two copies of the plasmid carrying the $X I$ gene were integrated into the genome, leading to higher $X I$ expression levels in the yeast cell cytoplasm. This also indicates that rapid xylose consumption requires a synergistic interaction resulting from different promoters, driving high $X I$ activity in the genome, 

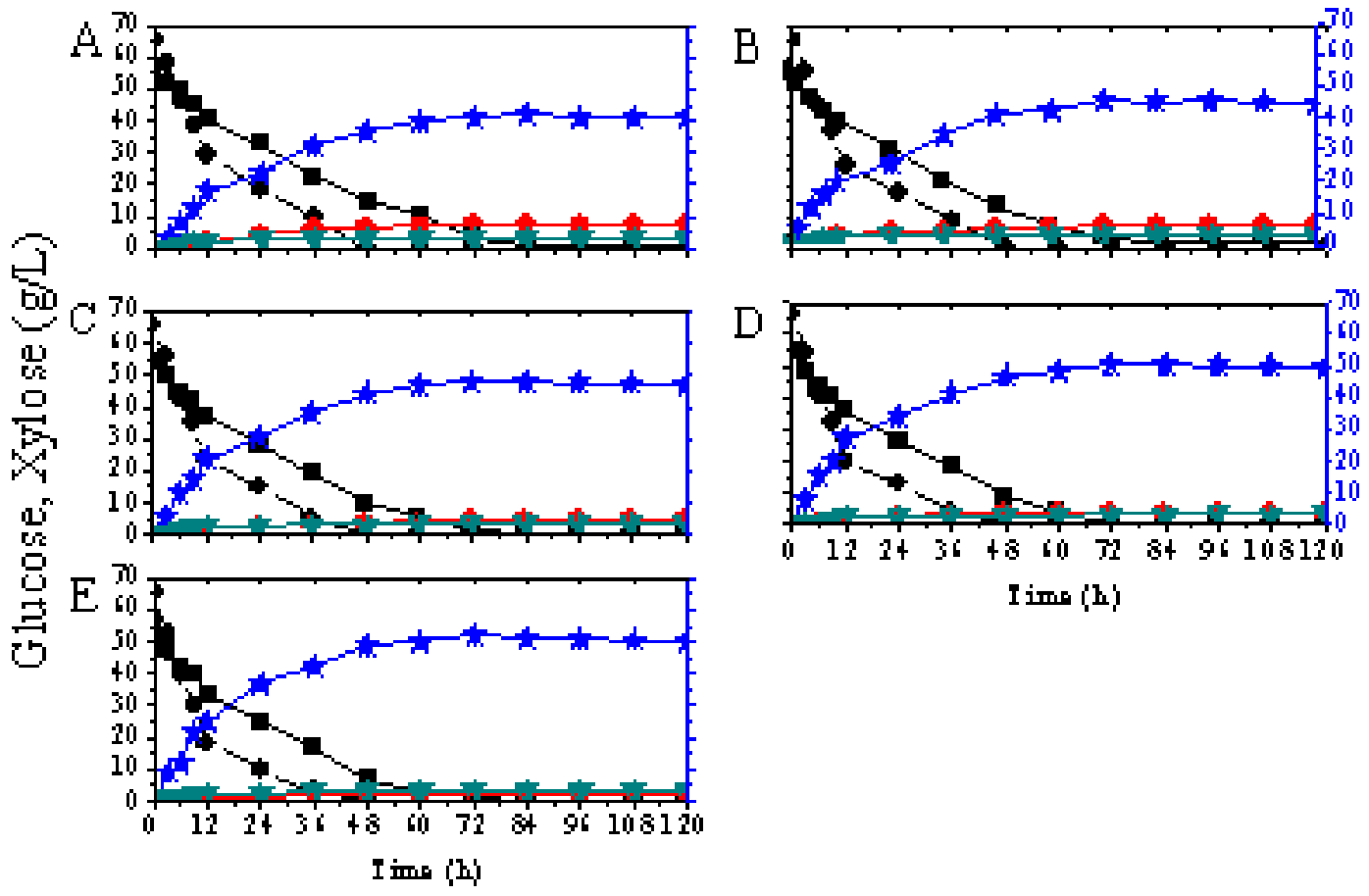

Figure 2. Time-dependent ethanol fermentation profiles of the engineered strains CW2 (A), CW3 (B), CW4 (C), CW5 (D), and CW6 (E) in YPDX medium with $55 \mathrm{~g} / \mathrm{L}$ xylose and $65 \mathrm{~g} / \mathrm{L}$ glucose. Symbols: black squares, xylose; black circles, glucose; red circles, xylitol; blue stars, ethanol; cyan triangles, biomass. Error bars represent the standard deviations from triplicate experiments.

Table 4.Fermentation profile of five $X I$ strains, CW2-6.

\begin{tabular}{lccccc}
\hline Parameters & CW2 & CW3 & CW4 & CW5 & CW6 \\
\hline $\begin{array}{l}\text { Initial glucose } \\
\text { concentration (g/L) }\end{array}$ & $65.5 \pm 0.01$ & $65.5 \pm 0.02$ & $65.2 \pm 0.00$ & $65.4 \pm 0.01$ & $65.5 \pm 0.01$ \\
$\begin{array}{l}\text { Initial xylose } \\
\text { concentration (g/L) }\end{array}$ & $54.5 \pm 0.01$ & $54.5 \pm 0.00$ & $54.6 \pm 0.01$ & $54.7 \pm 0.01$ & $54.5 \pm 0.02$ \\
$\begin{array}{l}\text { Maximum ethanol } \\
\text { concentration (g/L) }\end{array}$ & $42.2 \pm 0.02$ & $45.5 \pm 0.01$ & $45.8 \pm 0.01$ & $46.5 \pm 0.00$ & $47.9 \pm 0.01$ \\
$\begin{array}{l}\text { Specific glucose consumption } \\
\text { rate (g/g DCW/h) }\end{array}$ & $1.86 \pm 0.01$ & $1.98 \pm 0.02$ & $2.43 \pm 0.01$ & $2.61 \pm 0.00$ & $3.25 \pm 0.01$ \\
$\begin{array}{l}\text { Specific xylose consumption } \\
\text { rate (g/g DCW/h) }\end{array}$ & $0.71 \pm 0.00$ & $0.74 \pm 0.01$ & $0.90 \pm 0.02$ & $0.95 \pm 0.01$ & $1.10 \pm 0.00$ \\
$\begin{array}{l}\text { Specific ethanol production } \\
\text { rate (g/g DCW/h) }\end{array}$ & $1.08 \pm 0.00$ & $1.12 \pm 0.01$ & $1.35 \pm 0.00$ & $1.36 \pm 0.01$ & $1.42 \pm 0.02$ \\
$\begin{array}{l}\text { Ethanol yield } \\
\text { (g/g total sugar) }\end{array}$ & $0.35 \pm 0.00$ & $0.38 \pm 0.01$ & $0.38 \pm 0.00$ & $0.39 \pm 0.02$ & $0.4 \pm 0.01$ \\
$\begin{array}{l}\text { Xylitol yield } \\
\text { (g/g total sugar) }\end{array}$ & $0.07 \pm 0.00$ & $0.05 \pm 0.01$ & $0.05 \pm 0.00$ & $0.05 \pm 0.01$ & $0.04 \pm 0.00$ \\
\hline
\end{tabular}


Table 5.Fermentation profile of XI strains CW7, CW8, and CW9.

\begin{tabular}{lccc}
\hline Parameters & CW7 & CW8 & CW9 \\
\hline $\begin{array}{l}\text { Initial glucose } \\
\text { concentration }(\mathrm{g} / \mathrm{L})\end{array}$ & $65.5 \pm 0.02$ & $65.5 \pm 0.01$ & $65.5 \pm 0.01$ \\
$\begin{array}{l}\text { Initial xylose } \\
\text { concentration (g/L) }\end{array}$ & $54.5 \pm 0.00$ & $54.4 \pm 0.02$ & $54.5 \pm 0.01$ \\
$\begin{array}{l}\text { Maximum ethanol } \\
\text { concentration (g/L) }\end{array}$ & $50.2 \pm 0.02$ & $51.8 \pm 0.01$ & $54 \pm 0.01$ \\
$\begin{array}{l}\text { Specific glucose consumption } \\
\text { rate }(\mathrm{g} / \mathrm{g} \text { DCW/h) }\end{array}$ & $3.31 \pm 0.02$ & $3.43 \pm 0.00$ & $3.58 \pm 0.01$ \\
$\begin{array}{l}\text { Specific xylose consumption } \\
\text { rate }(\mathrm{g} / \mathrm{g} \text { DCW/h) }\end{array}$ & $1.26 \pm 0.00$ & $1.29 \pm 0.01$ & $1.34 \pm 0.01$ \\
$\begin{array}{l}\text { Specific ethanol production } \\
\text { rate }(\mathrm{g} / \mathrm{g} \text { DCW/h) }\end{array}$ & $1.43 \pm 0.00$ & $1.55 \pm 0.02$ & $1.76 \pm 0.01$ \\
$\begin{array}{l}\text { Ethanol yield (g/g total sugar) } \\
\text { Xylitol yield (g/g total sugar) }\end{array}$ & $0.42 \pm 0.01$ & $0.43 \pm 0.00$ & $0.45 \pm 0.01$ \\
\hline
\end{tabular}

which is consistent with a previous report showing that high xylose utilization could only be partially attributed to the high activity of XI (Zhou et al., 2012).

\subsection{Anaerobic Fermentation of the Improved Strains Expressing $X I$ in the Cell Plasma Membrane}

Next, we evaluated the fermentation profiles of three continuous XI strains (CW7-9) located in the cell plasma membrane (PM), in comparison with the parent strain CW6, located in the cell cytoplasm, based on the transporter locations and copy number variations of $X I$ expression. Co-fermentations in rich YP medium containing $65 \mathrm{~g} / \mathrm{L}$ glucose and $55 \mathrm{~g} / \mathrm{L}$ xylose were used to compare the performance of strains CW7-9 established through sequential metabolic/evolutionary engineering steps (Figure 3). The three engineered PM XI strains CW7-9 partially consumed $65 \mathrm{~g} / \mathrm{L}$ glucose after $36 \mathrm{~h}$, and completely consumed $55 \mathrm{~g} / \mathrm{L}$ xylose after $72 \mathrm{~h}$, producing high ethanol yields $(0.42 \mathrm{~g} / \mathrm{g}, 0.43 \mathrm{~g} / \mathrm{g}$, and $0.45 \mathrm{~g} / \mathrm{g}$ total sugar, respectively) and low xylitol yields $(0.04 \mathrm{~g} / \mathrm{g}, 0.02$ $\mathrm{g} / \mathrm{g}$, and $0.02 \mathrm{~g} / \mathrm{g}$ total sugar, respectively) (Figure $3 \mathrm{~A}-\mathrm{C}$ and Table 5). The PM strain CW8 showed higher ethanol production compared with $\mathrm{CW7}$, suggesting that $\mathrm{CW} 8$, harboring the endogenous fusion protein $X I \& \mathrm{XI}^{6 m}$, had slightly faster glucose and xylose consumption rates compared to CW7, harboring the exogenous fusion protein $G X S 1 \& \mathrm{XI}^{6 m}$ (Figure 3A, 3B and Table 5). Strains CW8 and CW9 showed an improved maximum sugar consumption rate and ethanol production rate compared to CW7, respectively (Table 5). The strain showing the best performance in this study (CW9) consumed all of the glucose and more than $99.7 \%$ of the xylose in approximately $72 \mathrm{~h}$, with an ethanol yield of $0.45 \mathrm{~g} / \mathrm{g}$ total sugar, equivalent to $90 \%$ of the maximum theoretical ethanol yield (Figure 3C and Table 5).

Although CW6 showed improved xylose consumption and

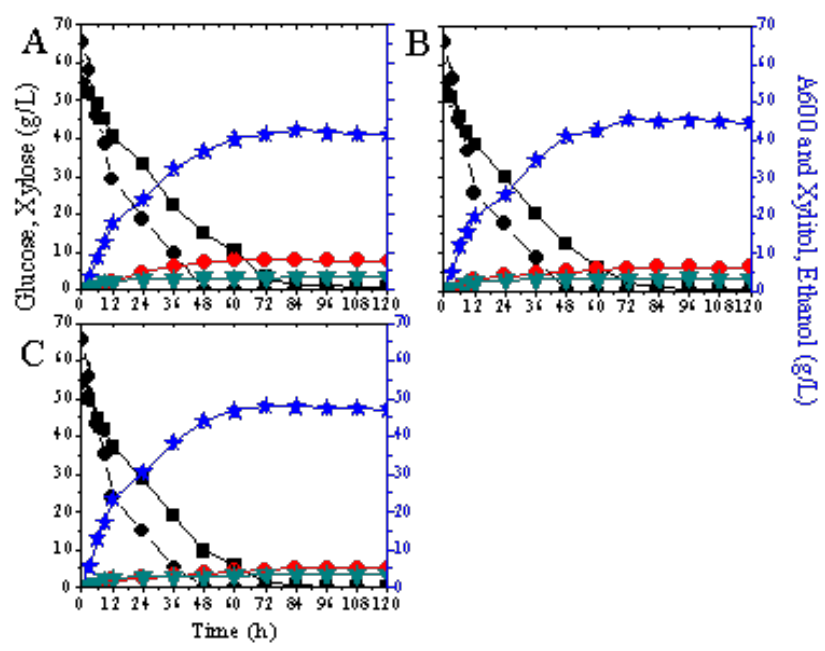

Figure 3. Time-dependent ethanol fermentation profiles of engineered strains CW7 (A), CW8 (B), and CW9 (C) in YPDX medium with $55 \mathrm{~g} / \mathrm{L}$ xylose and $65 \mathrm{~g} / \mathrm{L}$ glucose. Symbols: black squares, xylose; black circles, glucose; red circles, xylitol; blue stars, ethanol; cyan triangles, biomass. Error bars represent the standard deviations from triplicate experiments.

ethanol production by expressing two copies of the mutant $X I$ gene in the cell cytoplasm compared to strains CW2-5, a certain amount of xylose was still left unfermented, and the rate of xylose utilization by strain CW6 was too low to achieve viable ethanol production. Therefore, obtaining a faster xylose consumption rate and ethanol production rate could be expected through additional metabolic/evolutionary engineering. The yield of ethanol obtained with PM strain CW8 derived from strain CW6 was slightly higher than that obtained with the similar PM strain CW7. Therefore, the sustained improvement in the rate of xylose fermentation and ethanol production for CW8 and CW7 might be explained by the presence of a suitable combination of an important fusion protein with XI introduced by a xylose transporter gene such as endogenous HXT7 or exogenous GXS1.

By overexpressing two additional copies of the mutated $X I$ gene in the PM for CW9, we demonstrated that the gradual engineering of mutated $X I$ through exploitation of the two different fusion protein strategies described above could lead to the highest xylose-to-ethanol conversion yield. The improved enzyme activity was observed from the six mutations of the XI gene (E15D, E114G, E129D, T142S, A177T, and V433I). It seems plausible that the molecular transport of xylose into the cell, upon fast uptake depending on the expressed transporters $H X T 7$ or $G X S 1$, first aggregates into the dimer, which is partially active. This dimer can then associate into the tetramer, resulting in the fusion of XIs forming the ordered dimer/tetramer structure. This observation is also likely to be due to the effects of some mutations near the monomer-binding sites. Basically, fusing the xylose uptake by the expressed transporters and xylose utilization by subsequent $X I$ appears to be a rational spatial arrangement that can be used to quickly and effectively rewire xylose metabolism. The improved XI reported herein could serve as a critical starting point for further strain engineering. It 
is likely that a combination of the strong promoter of $P G K 1$ and the glucose/xylose transporter promoter of $H X T 7$ in the cell cytoplasm work together to regulate mutated $X I$ to boost ethanol yields and productivity. Moreover, other exogenous, endogenous, or intrinsic xylose transporters in the cell PM could jointly improve xylose uptake and utilization efficiency. Thus, the rapid fermentation and complete sugar attenuation observed was likely due to the high level of xylose uptake. Xylose transport was also possibly improved because of the combined effect of the xylose transporter with mutated XI, which is consent with the results of a recent study (Thomik et al., 2017). Therefore, the improvement of xylose transport is likely attributed to changes in the transporter expression level.

Zhou et al. (2012) confirmed that a high expression level of $X I$ could significantly promote a rapid xylose assimilation rate. Their engineered strains exhibiting high xylose assimilation rates harbored a large number of $X I$ gene copies, which is consistent with the high transcription level and enzymatic activity of $X I$. In the present study, the increase in the ethanol yield for CW6 or CW9 was in fact slightly higher than that expected from xylose conversion alone for CW2/CW3/CW4/CW5 or CW7/CW8, respectively. However, Demeke et al. (2007) inferred that high XI activity was likely the main, but not the only, reason for fast xylose assimilation capacity. The xylose consumption rate and ethanol production rate of our optimized strain CW9 were improved by moderately overexpressing four copies of a mutated $X I$ gene, without imposing a greater metabolic burden on the xylose assimilation pathway. This indicates that achieving both rapid xylose consumption and high ethanol production requires a synergistic interaction between high $X I$ expression levels and appropriate $X I$ mutation in the genome. In summary, we successfully optimized the PPP genes involved in the xylose utilization pathway (downstream approach), and introduced an efficient xylose transporter to fuse with a mutated XI gene (upstream approach) to further improve the xylose fermentation efficiency of engineered $S$. cerevisiae.

\section{Conclusion}

We describe the establishment of optimized strains of the yeast $S$. cerevisiae to improve $X I$ uptake using an evolutionary engineering approach. Specifically, this strategy involves the introduction of specific mutations in the XI gene with fusion of exogenous promoters during fermentation. The results showed that the best-performing strain CW9 improved XI uptake to increase ethanol production at $90 \%$ of the theoretical yield. The strategy proposed and results obtained demonstrate the value of targeting the xylose assimilation pathway for enhancing biofuel production in engineered yeast strains. The improved XI reported herein could serve as a critical starting point for further strain engineering and help increase bioethanol production in a cost-effective manner.

\section{Funding}

This work was financially supported by the National Natural Science Foundation of China (NSFC) (No. 31570044) and the Natural Science Foundation of Beijing Municipal Commission of Education (Grant No. KM201710028011).

\section{Author contributions}

Mei Zhang and Wen-Jing Fan performed this study. Jing-Yu Wang and Li-Min Cao designed this experiment. Li-min Cao wrote this manuscript.

\section{Conflict of Interest}

No conflict of interest was reported by all authors.

\section{Electronic Supplementary Materials}

This article contains supplementary materials in the following website:

http://ojs.udspub.com/index.php/aeb/rt/suppFiles/462/0

\section{References}

Apel, A.R., Ouellet, M., Szmidt-Middleton, H., Keasling, J.D. and Mukhopadhyay, A., 2016. Evolved hexose transporter enhances xylose uptake and glucose/xylose co-utilization in Saccharomyces cerevisiae. Scientific Reports, 6, 19512. https://doi.org/10.1038/srep19512

Bengtsson, O., Hahn-Hägerdal, B. and Gorwa-Grauslund, M.F., 2009. Xylose reductase from Pichia stipitis with altered coenzyme preference improves ethanolic xylose fermentation by recombinant Saccharomyces cerevisiae. Biotechnology for Biofuels, 2(1), 9. https://doi.org/10.1186/1754-6834-2-9

Bond-Watts, B.B., Bellerose, R.J. and Chang, M.C., 2011. Enzyme mechanism as a kinetic control element for designing synthetic biofuel pathways. Nature Chemical Biology, 7(4), 222-227.

https://doi.org/10.1038/nchembio.537

Brat, D., Boles, E. and Wiedemann, B., 2009. Functional expression of a bacterial xylose isomerase in Saccharomyces cerevisiae. Applied and Environmental Microbiology, 75(8), 2304-2311. https://doi.org/10.1128/AEM.02522-08

Demeke, M.M., Dietz, H., Li, Y., Foulquié-Moreno, M.R., Mutturi, S., Deprez, S., Den Abt, T., Bonini, B.M., Liden, G., Dumortier, F. and Verplaetse, A., 2013. Development of a D-xylose fermenting and inhibitor tolerant industrial Saccharomyces cerevisiae strain with high performance in lignocellulose hydrolysates using metabolic and evolutionary engineering. Biotechnology for Biofuels, 6(1), 89. https://doi.org/10.1186/1754-6834-6-89

Eliasson, A., Christensson, C., Wahlbom, C.F. and Hahn-Hägerdal, B., 2000. Anaerobic xylose fermentation by recombinant Saccharomyces cerevisiae carrying XYL1, XYL2, and XKS1 
in mineral medium chemostat cultures. Applied and Environmental Microbiology, 66(8), 3381-3386.

https://doi.org/10.1128/AEM.66.8.3381-3386.2000

Hou, J., Shen, Y., Li, X.P. and Bao, X.M., 2007. Effect of the reversal of coenzyme specificity by expression of mutated Pichia stipitis xylitol dehydrogenase in recombinant Saccharomyces cerevisiae. Letters in Applied Microbiology, 45(2), 184-189. https://doi.org/10.1111/j.1472-765X.2007.02165.x

Jeffries, T.W., Grigoriev, I.V., Grimwood, J., Laplaza, J.M., Aerts, A., Salamov, A., Schmutz, J., Lindquist, E., Dehal, P., Shapiro, H., Jin, Y.S., Passoth, V. and Richardson, P.M., 2007. Genome sequence of the lignocellulose-bioconverting and xylose-fermenting yeast Pichia stipitis. Nature Biotechnology, 25(3), 319-326.

https://doi.org/10.1038/nbt1290

Johansson, B., Christensson, C., Hobley, T. and Hahn-Hägerdal, B., 2001. Xylulokinase overexpression in two strains of Saccharomyces cerevisiae also expressing xylose reductase and xylitol dehydrogenase and its effect on fermentation of xylose and lignocellulosic hydrolysate. Applied and Environmental Microbiology, 67(9), 4249-4255. https://doi.org/10.1128/AEM.67.9.4249-4255.2001

Kong, Q.X., Cao, L.M., Zhang, A.L. and Chen, X., 2007. Overexpressing GLT1 in gpd1Delta mutant to improve the production of ethanol of Saccharomyces cerevisiae. Applied Microbiology and Biotechnology, 73(6), 1382-1386. https://doi.org/10.1007/s00253-006-0610-4

Kuyper, M., Hartog, M.M., Toirkens, M.J., Almering, M.J., Winkler, A.A., van Dijken, J.P. and Pronk, J.T., 2005. Metabolic engineering of a xylose-isomerase-expressing Saccharomyces cerevisiae strain for rapid anaerobic xylose fermentation. FEMS Yeast Research, 5(4-5), 399-409. https://doi.org/10.1016/j.femsyr.2004.09.010

Lee, S.M., Jellison, T. and Alper, H.S., 2012. Directed evolution of xylose isomerase for improved xylose catabolism and fermentation in the yeast Saccharomyces cerevisiae. Applied and Environmental Microbiology, 78(16), 5708-5716. https://doi.org/10.1128/AEM.01419-12

Madhavan, A., Tamalampudi, S., Ushida, K., Kanai, D., Katahira, S., Srivastava, A., Fukuda, H., Bisaria, V.S. and Kondo, A., 2009. Xylose isomerase from polycentric fungus Orpinomyces: gene sequencing, cloning, and expression in Saccharomyces cerevisiae for bioconversion of xylose to ethanol. Applied Microbiology and Biotechnology, 82(6), 1067. https://doi.org/10.1007/s00253-008-1794-6

Nijland, J.G., Vos, E., Shin, H.Y., Waal, P.P., Klaassen, P. and Driessen, A.J., 2016. Improving pentose fermentation by preventing ubiquitination of hexose transporters in Saccharomyces cerevisiae. Biotechnology for Biofuels, 9(1), 158. https://doi.org/10.1186/s13068-016-0573-3

Olofsson, K., Runquist, D., Hahn-Hägerdal, B. and Lidén, G., 2011. A mutated xylose reductase increases bioethanol production more than a glucose/xylose facilitator in simultane-
Saloheimo, A., Rauta, J., Stasyk, O.V., Sibirny, A.A., Penttilä, M. and Ruohonen, L., 2007. Xylose transport studies with xylose-utilizing Saccharomyces cerevisiae strains expressing heterologous and homologous permeases. Applied Microbiology and Biotechnology, 74(5), 1041-1052.

https://doi.org/10.1007/s00253-006-0747-1

Shen, Y., Chen, X., Peng, B., Chen, L., Hou, J. and Bao, X., 2012. An efficient xylose-fermenting recombinant Saccharomyces cerevisiae strain obtained through adaptive evolution and its global transcription profile. Applied Microbiology and Biotechnology, 96(4), 1079-1091. https://doi.org/10.1007/s00253-012-4418-0

Subtil, T. and Boles, E., 2012. Competition between pentoses and glucose during uptake and catabolism in recombinant Saccharomyces cerevisiae. Biotechnology for Biofuels, 5(1), 14. https://doi.org/10.1186/1754-6834-5-14

Thomik, T., Wittig, I., Choe, J.Y., Boles, E. and Oreb, M., 2017. An artificial transport metabolon facilitates improved substrate utilization in yeast. Nature Chemical Biology, 13(11), 11581163. https://doi.org/10.1038/nchembio.2457

Xiong, M., Chen, G. and Barford, J., 2011. Alteration of xylose reductase coenzyme preference to improve ethanol production by Saccharomyces cerevisiae from high xylose concentrations. Bioresource Technology, 102(19), 9206-9215. https://doi.org/10.1016/j.biortech.2011.06.058

Young, E.M., Comer, A.D., Huang, H. and Alper, H.S., 2012. A molecular transporter engineering approach to improving xylose catabolism in Saccharomyces cerevisiae. Metabolic Engineering, 14(4), 401-411. https://doi.org/10.1016/j.ymben.2012.03.004

Yuan, T., Guo, Y., Dong, J., Li, T.Y., Zhou, T., Sun, K.W., Zhang, M., Wu, Q.Y., Xie, Z., Cai, Y.Z. and Cao, L.M., 2017. Construction, characterization and application of a genome-wide promoter library in Saccharomyces cerevisiae. Frontiers of Chemical Science and Engineering, 11(1), 107-116. https://doi.org/10.1007/s11705-017-1621-7

Zhou, H., Cheng, J.S., Wang, B.L., Fink, G.R. and Stephanopoulos, G., 2012. Xylose isomerase overexpression along with engineering of the pentose phosphate pathway and evolutionary engineering enable rapid xylose utilization and ethanol production by Saccharomyces cerevisiae. Metabolic Engineering, 14(6), 611-622.

https://doi.org/10.1016/j.ymben.2012.07.011

ous fermentation and co-fermentation of wheat straw. Amb Express, 1(1), 4. https://doi.org/10.1186/2191-0855-1-4

Salis, H.M., Mirsky, E.A. and Voigt, C.A., 2009. Automated design of synthetic ribosome binding sites to control protein expression. Nature Biotechnology, 27(10), 946-950. https://doi.org/10.1038/nbt.1568 